\section{Refractory Hypotension Relat- ed to Systemic Mastocytosis Diagnosed After Cytoreductive Therapy for Acute Myeloid Leukemia}

\section{Denisa Ferastraoaru*1, Murali Janakiram ${ }^{2}$, Ronald Rice $^{3}$ and Golda Hudes ${ }^{1}$}

${ }^{1}$ Department of Medicine, Allergy and Immunology Division, Albert Einstein College of Medicine, Bronx, USA

${ }^{2}$ Department of Oncology, Albert Einstein College of Medicine, New York, USA

${ }^{3}$ Department of Pathology, Albert Einstein College of Medicine, New York, USA

\begin{abstract}
Systemic Mastocytosis (SM) can be associated with Acute Myeloid Leukemia (AML) and mast cells can be masked on bone marrow biopsy by the high burden of blast cells. Most of the SM-AML patients have associated translocation $\mathrm{t}(8 ; 21)$ and mutation of D816V KIT gene which were not present in the reported case. None of the SM-AML cases described in the literature had systemic symptoms secondary to SM. This is the first reported case of life-threatening symptomatic SM in a patient with SM-AML. Checking tryptase levels in symptomatic patients will lead to prompt initiation of mast cell mediators blocking therapy resulting in improved clinical management. Keywords: Acute myeloid leukemia; Negative c-kit mutation; Symptomatic systemic mastocytosis
\end{abstract}

\section{Abbrevations}

AML: Acute Myeloid Leukemia; MC: Mast Cells; SM: Systemic Mastocytosis; SM-AML: Systemic Mastocytosis Associated With Acute Myeloid Leukemia

\section{Introduction}

Systemic Mastocytosis (SM) can be Aassociated with Clonal Hematological Non Mast Cell Lineage Disorders (AHNMD) like Acute Myeloid Leukemia (AML) or Myelodysplastic Syndrome (MDS) in up to $10-40 \%$ of patients and carries a poor prognosis [1-2]. Most of the

*Corresponding author: Denisa Ferastraoaru, Department of Medicine, Allergy and Immunology Division, Albert Einstein College of Medicine, Bronx, USA, Tel: +1 6462750584; E-Mail: dferastr@montefiore.org

Citation: Ferastraoaru D, Janakiram M, Rice R, Hudes G (2017) Refractory Hypotension Related to Systemic Mastocytosis Diagnosed After Cytoreductive Therapy for Acute Myeloid Leukemia. J Allergy Disord Ther 3: 006.

Received: January 16, 2017; Accepted: April 17, 2017; Published: May 02, 2017
SM-AML cases have associated translocation $\mathrm{t}(8 ; 21)$ and mutation of D816V KIT gene [2]. Here we present a patient with SM-AML without translocation $\mathrm{t}(8 ; 21)$ and D816V KIT mutation who developed refractory hypotension due to Mast Cells (MC) mediators release after blastolytic therapy. In this case, the diagnosis of SM was made after cytoreductive therapy for AML.

\section{Clinical Case}

The patient is 53-year-old female who was initially diagnosed with myeloproliferative-myelodysplastic syndrome which subsequently progressed to high risk MDS with complex cytogenetics (deletion of chromosomes 5, 7 and trisomy 8). She received standard induction chemotherapy with cytarabine and idarubicin. Four weeks later she developed urticaria that was thought to be in relation to one of the prophylaxis medications (fluconazole, valacyclovir or mepron). However the urticaria slowly resolved over a month with daily antihistamines despite continuing all three medications.

One month after this episode she started to develop again pancytopenia. The bone marrow biopsy was repeated and showed conversion to Acute Myeloid Leukemia (AML) with 21\% blasts for which re-induction chemotherapy with High Dose Cytarabine (HiDAC) was initiated. The bone marrow biopsy revealed same cytogenetics as before: deletion of chromosomes 5, 7 and trisomy 8 .

Hospital course was complicated by neutropenic fevers for which she received empiric antibiotic coverage and symptomatic hypotension (nadir of systolic blood pressure of $75 \mathrm{mmHg}$ ) responsive to fluids. Clinically, the patient was stable, however, she developed new diffuse and persistent pruritic maculo-erythematous rash (skin biopsy showed spongiosis) which was thought to be medication related. Fifteen days after HiDAC initiation she developed vancomycin resistant E. faecium bacteremia treated with daptomycin subsequently with negative blood cultures. After two weeks, despite the appropriate antimicrobial treatment, the patient started to have severe episodes of hypotension non responsive to fluids resuscitation and eventually required intubation and initiation of three vasopressorswhich were difficult to taper. Extensive work-up in the intensive care unit excluded cardiac and septic shocks as causes for refractory hypotension. A repeat Bone Marrow (BM) biopsy to evaluate response to chemotherapy revealed clearance of myeloid blasts but showed 20\% CD $117^{+}$ $\mathrm{CD} 2^{+} \mathrm{CD} 25$ oval spindle-shaped mast cells (Figure 1), which were positive for $\mathrm{CD} 117$ (Figure 2). Whole BM real time-polymerase chain reaction was negative for D816V KIT mutation. Serum tryptase level was $42 \mathrm{ng} / \mathrm{ml}$ (normal $<11.4 \mathrm{ng} / \mathrm{ml}$ ). The patient was diagnosed with SM-AML and started on histamine-1, histamine-2 and leukotriene receptors blockers with significant improvement in blood pressure (able to taper the vasopressors) and skin rash. Unfortunately, the patient progressively developed acidosis in relation to acute kidney injury secondary to prolonged hypotension, then multi-organ failure and died 2 weeks later. 


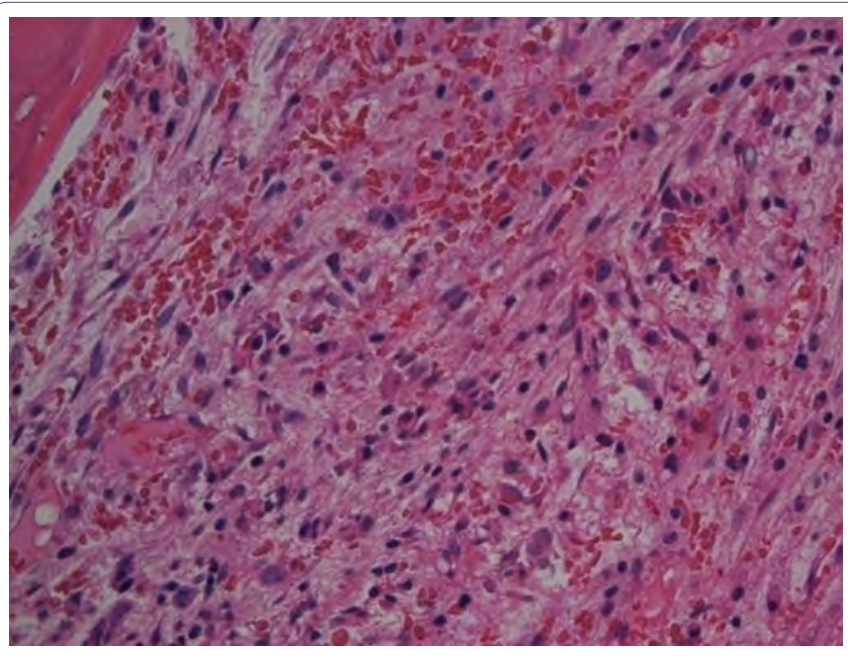

Figure 1: Hypercellular Bone Marrow with Atypical Mast Cells.

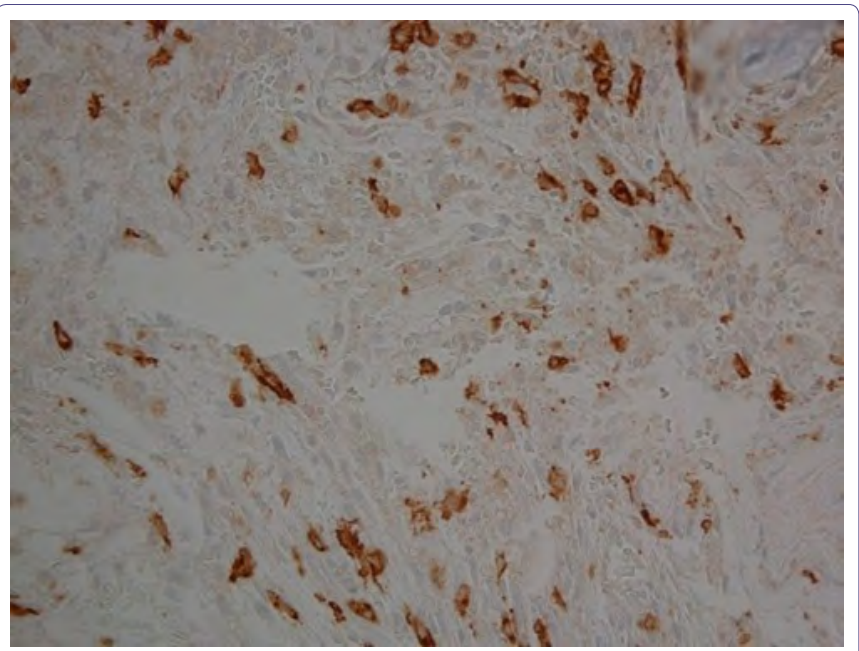

Figure 2: CD117+ Mast Cells in the Bone Marrow.

\section{Discussion}

Systemic mastocytosis is a myeloproliferative neoplasm characterized by infiltration with MC of different tissues. Mast cells are recognized by production of tryptase and expression of different surface markers such as CD25, CD2 and CD117 (KIT). CD 117(KIT) is a tyrosine kinase receptor encoded by the c-kit proto-oncogene. Mutation in the KIT codon 816 (D816V) is associated with autonomous growth of MC and is detectable in vast majority of patients with SM [3]. KIT D816V mutation represents one of the minor criteria for diagnosis of $\mathrm{SM}$, but its absence does not exclude the diagnosis.

Our case is unique due to several characteristics.

First, the diagnosis of SM-AML was made after cytoreductive chemotherapy for AML. There have been published few cases of SM being diagnosed after treatment for AML [4-6] but none of those patients had systemic symptoms secondary to SM. In our case there was a clear evidence of SM - induced refractory hypotension because of negative work-up for other causes and improvement in blood pressure after blocking therapy of $\mathrm{MC}$ mediators was initiated. Moreover, the patient had two episodes of pruritic skin rash which we suspect that probably were in relation to MC mediators release. The skin biopsy result was nonspecific (spongiosis), however, special stain for mast cells was not performed at the time of skin biopsy. Unfortunately, after the patient's death, it was not possible to re-examine the old skin and bone marrow biopsies to retrospectively look for the presence of mast cells. The diagnosis of SM in this patient was made on the basis of one major criterion ( $\mathrm{MC}$ aggregates in the $\mathrm{BM}$ ) and one minor criterion (presence of spindle-shaped atypical MC with aberrant expression of CD2 in the BM). Coexistence of SM with AML lead to final diagnosis of SM-AML.

It is not unusual to diagnose SM after AML treatment because the initial high burden of blasts is thought to cover the atypical MC [4-6] but the rapid diagnosis of associated SM has major impact if the patient is symptomatic due to MC mediator release. Our patient is the first reported case of life-threatening symptomatic SM-AML due to refractory hypotension that responded to medications blocking the effects of MC mediators.

Second, c-kit mutation D816V was not detectable in either BM cells or peripheral blood, despite literature showing that KIT D816V mutation in AML is highly associated with co-existing SM [2]. However, another mutation at this or another locus cannot be excluded. Fritsche-Polanz et al. investigated 101 patients with AML and found expression of KIT D816V in 6.9\%; all of these cases were found to have associated SM. D816V mutation has been associated with poor prognosis in SM and resistance to imatinib, but is unclear if its absence represents a prognostic factor [7].

Third, the patient did not have associated translocation $\mathrm{t}(8 ; 21)$ which was described in patients with SM-AML. The incidence of SM in patients with $t(8 ; 21)$ is not known, but its presence is reported to carry a dismal prognosis. The $t(8 ; 21)$ occurs in about $5 \%$ of all AMLs and c-kit mutations play a role in the development of overt $t(8 ; 21)$ in about $20 \%$ of cases [8]. With this case, we would like to highlight the importance of suspecting SM in AML patients without translocation $\mathrm{t}(8 ; 21)$.

It is imperative to pursue an early diagnosis of SM in clonal malignant hematological disorders because the life-threatening MC mediators-related episodes justify the need for adequate treatment. Since diagnosis of SM in hematological neoplasms can be missed initially due to high cellularity burden, we propose to use tryptase as a biochemical measure of $\mathrm{MC}$ activation in those patients who develop skin rashes and/ or unexplained refractory hypotension. Current guidelines recommend prompt treatment with histamineand leukotriens antagonists to improve symptoms of MC degranulation [9].

\section{Conclusion}

In conclusion, this is the first reported case of life-threatening refractory hypotension due to MC mediators release in a patient with SM-AML without translocation $\mathrm{t}(8 ; 21)$ and KIT D816V mutation. Prompt diagnosis and treatment is of major importance and improves SM-related symptoms. Further analysis is required to evaluate this phenotype with emphasis on clinical symptoms and prognosis.

\section{References}

1. Sperr WR, Horny HP, Valent P (2002) Spectrum of associated clonal hematologic non-mast cell lineage disorders occurring in patients with systemic mastocytosis. Int Arch Allergy Immunol 127: 140-142.

2. Fritsche-Polanz R, Fritz M, Sotlar K, Sperr WR, Manhalter C, Fodinger M, et al. (2010) High frequency of concomitant mastocytosis in patients with acute myeloid leukemia exhibiting the transforming KIT mutation D816V. Molecular Oncology 4: 335-346. 
3. Lim KH, Tefferi A, Lasho TL, Finke C, Patnaik M, et al. (2009) Systemic mastocytosis in 342 consecutive adults: survival studies and prognostic factors. Blood 113: 5727-5736.

4. Pullarkat VA, Bueso-Ramos C, Lai R, Kroft S, Wilson CS, et al. (2003) Systemic mastocytosis with associated clonal hematological non-mast-cell lineage disease: analysis of clinicopathologic features and activating c-kit mutations. Am J Hematol 73: 12-17.

5. Horny HP, Sotlar K, Sperr WR, Valent P (2004) Systemic mastocytosis with associated clonal haematological non-mast cell lineage diseases: a histopathological challenge. J Clin Pathol 57: 604-608.
6. Bernd HW, Sotlar K, Lorenzen J, Osieka R, Fabry U, et al. ( 2004 ) Acute myeloid leukaemia with $t(8 ; 21)$ associated with "occult" mastocytosis. Report of an unusual case and review of the literature. J Clin Pathol. 57: 324-328.

7. Verstovsek S (2013) Advanced systemic mastocytosis: the impact of KIT mutations in diagnosis, treatment, and progression. Eur J Haematol 90: 89-98.

8. Pullarkat ST, Pullarkat V, Kroft SH, Wilson CS, Ahsanuddin AN, et al. (2009) Systemic mastocytosis associated with $\mathrm{t}(8 ; 21)(\mathrm{q} 22 ; \mathrm{q} 22)$ acute myeloid leukemia. J Hematop 2: 27-33.

9. Pardanani A (2016) Systemic mastocytosis in adults: 2017 update on diagnosis, risk stratification and management. Am J Hematol 91: 1146-1159. 